\title{
Entropy of constant curvature black holes in general relativity
}

\author{
Jolien D. E. Creighton \\ Theoretical Astrophysics, California Institute of Technology, Pasadena, California 91125 \\ Robert B. Mann \\ Department of Physics, University of Waterloo, Waterloo, Ontario, Canada N2L 3G1
}

(Received 21 October 1997; published 24 June 1998)

\begin{abstract}
We consider the thermodynamic properties of the constant curvature black hole solution recently found by Banados. We show that it is possible to compute the entropy and the quasilocal thermodynamics of the spacetime using the Einstein-Hilbert action of general relativity. The entropy we obtain is not associated with the event horizon; rather it is associated with the Killing horizon of static observers, which is tangent to the event horizon. This unusual feature of the constant curvature black hole has not been seen in other black hole spacetimes. [S0556-2821(98)00216-1]

PACS number(s): 04.70.Dy, 04.20.Ha, 04.70.Bw
\end{abstract}

It is generally believed that black holes have a fundamental role to play in furthering our understanding of the quantization of gravity. Indeed, a wide variety of spacetimes representing black holes with unusual properties has been discovered in the past decade as a consequence of an intensive study of the various approaches to quantum gravity. Further progress will necessarily entail a more thorough investigation of the basic thermodynamics of the different species of black holes.

A new type of black hole solution has been recently found by Bañados [1]. This solution, which is one possible generalization of the $(2+1)$-dimensional black hole [2] to higher dimensions, represents a black hole in a spacetime with toroidal topology $\left(\mathfrak{R}^{3} \times S^{1}\right)$ and constant curvature. The constant curvature black hole $(\mathrm{CCBH})$ is an anti-de Sitter spacetime with identifications, and so it is a solution of any theory that contains anti-de Sitter spacetimes. ${ }^{1}$

In this paper, we examine the thermodynamic properties of the CCBH spacetime in general relativity. In general, a given black hole solution can arise from a variety of theories, and its thermodynamic properties are theory-dependent. In order to understand the thermodynamic properties of CCBHs, Bañados considered the black hole to be a solution of a five-dimensional Chern-Simons supergravity theory. In such a theory, the thermodynamic variables can be constructed for a rotating solution, but the result is surprising: the thermodynamic internal energy is associated with the angular momentum parameter of the solution while the thermodynamic conjugate to the angular velocity is associated with the mass parameter. In addition, the entropy is found to be proportional to the circumference of the inner horizon rather than the outer horizon. Such phenomena also occur for the $(2+1)$-dimensional black hole when the thermodynamic variables are computed from a Chern-Simons like action [4], though a more conventional result for the thermodynamic

\footnotetext{
${ }^{1}$ An examination of all identifications in four dimensional anti-de Sitter spacetime has been presented by Holst and Peldán [3]; the $\mathrm{CCBH}$ manifold described by Bañados can be considered to be a submanifold of one of the solutions found in Ref. [3].
}

variables is obtained when the action of general relativity is used [5]. We consider here CCBHs in the context of fourdimensional general relativity, although our results may be straightforwardly generalized to any larger number of dimensions. $^{2}$

For definiteness, we consider the non-rotating $\mathrm{CCBH}$ spacetime. This spacetime has the line element

$$
d s^{2}=\frac{\ell^{4} f^{2}(r)}{r_{\mathrm{H}}^{2}}\left[d \theta^{2}-\sin ^{2} \theta(d t / \ell)^{2}\right]+\frac{d r^{2}}{f^{2}(r)}+r^{2} d \phi^{2}
$$

with the metric function

$$
f^{2}(r)=\frac{r^{2}-r_{\mathrm{H}}^{2}}{\ell^{2}}
$$

The quantity $r_{\mathrm{H}}$ is the circumferential radius of the "bolt" of the event horizon, and $\ell$ is the length scale of the anti-de Sitter spacetime curvature. The angle $\phi$ is periodic with period $2 \pi$; the coordinate system is valid outside the black hole (i.e., for $r>r_{\mathrm{H}}$ ) and for $0<\theta<\pi$. The details of the construction of this spacetime from an ordinary anti-de Sitter spacetime can be found in Ref. [1]. Because this solution is merely an anti-de Sitter spacetime with identifications, it is a solution to the field equations arising from the EinsteinHilbert action

$$
I=\int_{M} \boldsymbol{L}=\frac{1}{16 \pi} \int_{M}^{4} \boldsymbol{\epsilon}(R-2 \Lambda),
$$

with a cosmological constant $\Lambda=-3 / \ell^{2}$. Here, $\boldsymbol{L}$ is the Einstein-Hilbert Lagrangian 4-form (with a cosmological constant) and ${ }^{4} \boldsymbol{\epsilon}$ is the volume form on the manifold $M$.

The Lorentzian black hole spacetime is depicted in Fig. 1. Notice that the foliation of the spacetime into leaves of constant coordinate time $t$ is degenerate on the axis $A$ with $\theta$

\footnotetext{
${ }^{2}$ The thermodynamics of other asymptotically anti-de Sitter black holes with non-trivial spatial topology has been studied in Ref. [6].
} 


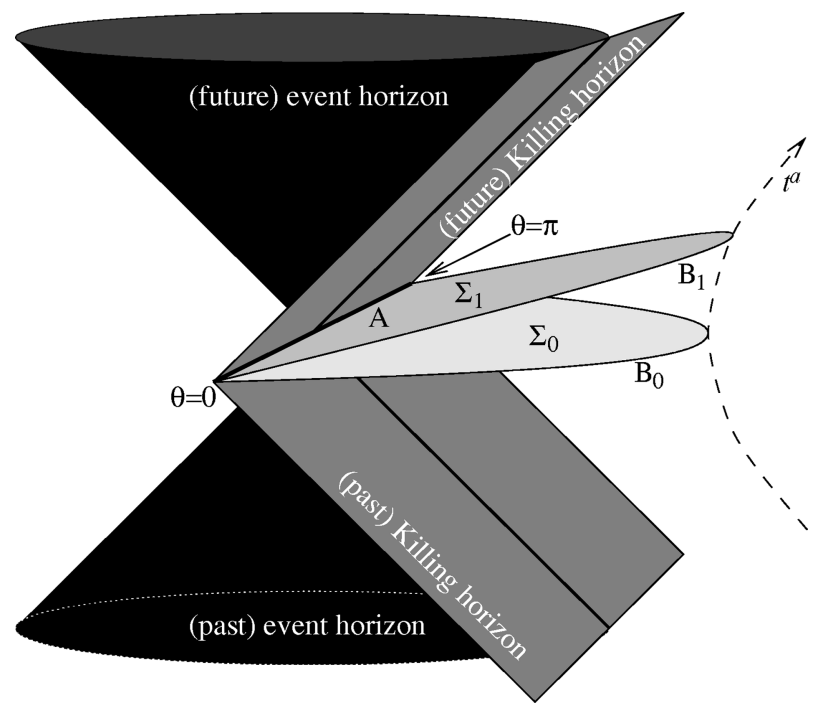

FIG. 1. The Lorentzian CCBH spacetime. The two cones represent the future and past event horizons of the black hole, while the spacelike surfaces $\Sigma_{0}$ and $\Sigma_{1}$ are surfaces of constant coordinate time. The singularity within the event horizon is not shown. Each point represents a circle in the suppressed coordinate $\phi$. The outer boundaries of $\Sigma_{0}$ and $\Sigma_{1}$ are the quasilocal surfaces of constant time and radius. The foliation is degenerate along the axis $A$, which is the bifurcation surface of the Killing horizon of the vector field $t^{a}$. The Killing horizon touches the event horizon along the thick dark lines in the middle of the Killing horizon.

$=0$ and $\theta=\pi$. Thus, in addition to the event horizons, there is a Killing horizon on which the Killing vector $t^{a}$ $=(\partial / \partial t)^{a}$ becomes null. ${ }^{3}$ It is an interesting feature of the $\mathrm{CCBH}$ spacetime that the Killing horizon of static observers of the black hole does not coincide with the event horizon of the black hole except along a particular surface (which is indicated by the thick dark lines in the middle of the Killing horizon in Fig. 1). Note that along this surface the event horizon and the Killing horizon share a generator. We will show that, for these static observers, the entropy is associated with the bifurcation surface of the Killing horizon. The quasilocal surface $B$ is taken to be a 2-surface of constant time and radius $r=R>r_{\mathrm{H}}$.

Let us begin our analysis of the properties of the $\mathrm{CCBH}$ with a calculation of the entropy. The entropy of a black hole spacetime is equal to the value of the microcanonical action of the Euclidean section of the spacetime [7]. In the case of the $\mathrm{CCBH}$, the Euclidean section is obtained by the Wick rotation $t \rightarrow \tau=i t$. The line element is

$$
d s^{2}=\frac{\ell^{4} f^{2}(r)}{r_{\mathrm{H}}^{2}}\left[d \theta^{2}+\sin ^{2} \theta(d \tau / \ell)^{2}\right]+\frac{d r^{2}}{f^{2}(r)}+r^{2} d \phi^{2} .
$$

\footnotetext{
${ }^{3}$ If a different static Killing vector had been chosen, there would be some other axis along which the foliation into leaves of constant coordinate time would become degenerate. We have assumed some arbitrary static Killing vector and chosen the coordinate $\theta$ such that the axis has $\theta=0$ and $\theta=\pi$.
}

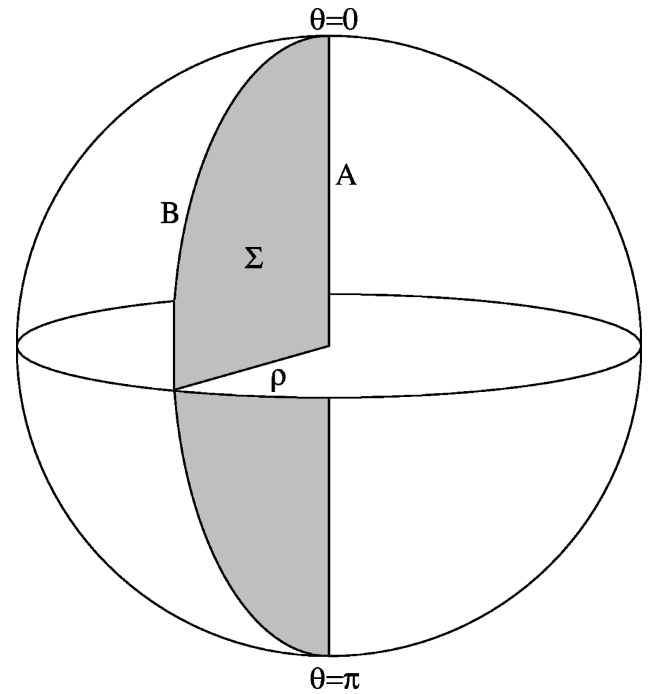

FIG. 2. The Euclidean CCBH instanton. The azimuthal angle is the time $\tau / \ell$, the polar angle is $\theta$, and the radius is the proper radius $\rho=\int d r / f(r)$ with $\rho\left(r_{\mathrm{H}}\right)=0$. Each point represents a circle in the suppressed coordinate $\phi$. The surface $\Sigma$ is a surface of constant time; its boundary consists of the quasilocal surface $B$ and the axis $A$ of the sphere.

Notice that the quantity in brackets is the line element of a two sphere if $0 \leqslant \theta \leqslant \pi$ and $\tau$ is periodic with period $2 \pi \ell$. If such an identification of the time is made, the Euclidean manifold is regular and is depicted in Fig. 2.

The microcanonical action differs from the action of Eq. (3) by a boundary term on the history $T$ of the quasilocal surface $B[8]$,

$$
I_{\text {micro }}=\int_{M} \boldsymbol{L}-\int_{T} d t \wedge q[t] .
$$

The boundary functional contains the Noether charge 2form, $q[t]$, associated with the covariance of the Lagrangian under diffeomorphisms generated by the vector $t^{a}=(\partial / \partial t)^{a}$ [9]. On a two-dimensional submanifold with binormal $n^{a b}$ and volume element ${ }^{2} \epsilon_{a b}=\frac{1}{2}{ }^{4} \boldsymbol{\epsilon}_{a b c d} n^{c d}$, the Noether charge 2 -form is given by

$$
\boldsymbol{q}[t]=\frac{1}{16 \pi}{ }^{2} \boldsymbol{\epsilon} n^{a b} \nabla_{a} t_{b} .
$$

The microcanonical action can be evaluated on the Euclidean manifold to yield the entropy. We follow the method of Iyer and Wald [8] in computing the entropy. Because the spacetime is stationary, we find

$$
S=\Delta \tau\left[-\int_{\partial \Sigma} \boldsymbol{q}[t]+\int_{B} \boldsymbol{q}[t]\right],
$$

with $\Delta \tau=2 \pi \ell$. From Fig. 2 , it is clear that $\partial \Sigma$ contains two pieces: the quasilocal surface $B$ and the axis $A$ of the spherical instanton. Thus, the entropy only depends on the integral

\footnotetext{
${ }^{4}$ The period of identification satisfies the usual regularity condition $\Delta \tau=2 \pi / \kappa$ with $\kappa=\left[-\frac{1}{2}\left(\nabla^{a} t^{b}\right)\left(\nabla_{a} t_{b}\right)\right]^{1 / 2}=1 / \ell$ evaluated on the Killing horizon.
} 
of the Noether charge 2-form over the axis $A$ of the spherical instanton. For a fixed time, the binormal to $A$ is $n^{a b}$ $=2 u^{[a} \mathrm{m}^{b]}$ (evaluated on the axis) where $u^{a}$ is the normal vector to the surface of constant time and $m^{a}$ is the normal vector to surfaces of constant $\theta$. The Noether charge 2-form is found to be $\boldsymbol{q}[t]={ }^{2} \boldsymbol{\epsilon}(8 \pi \ell)^{-1}$ where ${ }^{2} \boldsymbol{\epsilon}$ is the area element of the 2-surface $A$. Integrating the Noether charge over the boundary $A$ (which consists of both the portion with $\theta$ $=0$ and $\theta=\pi$ ), we find that the entropy is

$$
S=\pi \ell^{2} f(R)
$$

where $r=R$ is the radius of the quasilocal surface $B$. Notice that the entropy depends on the size of the quasilocal surface: this dependence occurs because the entropy is associated with the area of the cylinder $A$ which extends to $r$ $=R$.

We can also calculate the quasilocal thermodynamic variables in order to verify that the first law of thermodynamics holds. The relevant quantities we need are the quasilocal energy density and the surface stress tensor. These variables are calculated using the definitions of Brown and York [10]. The quasilocal energy density derived from the EinsteinHilbert action is given by

$$
\mathcal{E}=\frac{1}{8 \pi} \sqrt{\sigma} k
$$

Here, $k$ is the trace of the extrinsic curvature $k_{a b}$ of the quasilocal surface $B$ embedded in the spacelike surface $\Sigma$ : $k_{a b}=-\sigma_{a}^{c} D_{c} n_{b}$ where $D_{a}$ is the covariant derivative operator on $\Sigma, n^{a}$ is the normal vector to $B$ embedded in $\Sigma$, and $\sigma_{a b}$ is the induced metric on $B$. Similarly, the quasilocal surface stress tensor is

$$
\mathcal{S}^{a b}=\frac{1}{16 \pi} \sqrt{\sigma}\left[k^{a b}-\sigma^{a b}\left(k-n^{c} a_{c}\right)\right]
$$

where $a_{c}=u^{a} \nabla_{a} u_{c}$ is the acceleration of the timelike unit normal $u^{a}$ to the surfaces $B$ embedded in $T$. In general, the quasilocal energy density also has a contribution arising from an arbitrary background action functional; this contribution effectively provides a zero point for the energy in a reference spacetime. However, it is difficult to choose a reference spacetime for the CCBH because the intrinsic geometry of the quasilocal surface $B$ depends on the constant of integration $r_{\mathrm{H}}$. Fortunately, since the first law of thermodynamics only depends on changes in the quasilocal energy, the contribution from the reference spacetime is irrelevant when analyzing the thermodynamics of the spacetime.

The calculation of the quasilocal energy density and surface stress tensor is straightforward. From Eqs. (9) and (10), we obtain

$$
\mathcal{E}=-\frac{1}{8 \pi r_{\mathrm{H}}}\left[R^{2}+\ell^{2} f^{2}(R)\right]
$$

for the quasilocal energy density and

$$
\begin{aligned}
\mathcal{S}^{\theta \theta} & =\frac{1}{16 \pi} \frac{r_{\mathrm{H}}}{\ell^{4} f^{2}(R)}\left[R^{2}+\ell^{2} f^{2}(R)\right] \\
\mathcal{S}^{\phi \phi} & =\frac{1}{8 \pi r_{\mathrm{H}}}
\end{aligned}
$$

for the quasilocal stress tensor. In addition, the inverse temperature $\beta(R)$ on the quasilocal boundary can be computed: it is just the red-shifted period of identification of the Euclidean time. We find

$$
\beta(R)=\left[g_{t t}(R)\right]^{1 / 2} \Delta \tau=\frac{2 \pi \ell^{2} f(R)}{r_{\mathrm{H}}} \sin \theta .
$$

Notice that the temperature is not constant on the quasilocal surface. In particular, it diverges at $\theta=0$ and $\theta=\pi$. This is because the foliation becomes degenerate at these points.

The first law of thermodynamics is obtained by consideration of variations of the microcanonical action evaluated on the Euclidean manifold. As shown in Refs. [7, 8],

$$
\delta S=\int_{0}^{\pi} d \theta \int_{0}^{2 \pi} d \phi \beta\left[\delta \mathcal{E}+\mathcal{S}^{a b} \delta \sigma_{a b}\right] .
$$

Because the quasilocal boundary is not an isotherm, the first law of thermodynamics must be left in an integral form, i.e., the temperature cannot be factored out of the integral. Equation (14) can be explicitly verified using the quasilocal energy density of Eq. (11), the quasilocal stress tensor of Eq. (12), the temperature of Eq. (13) and the entropy of Eq. (8). Recall that $\sigma_{a b}$ is the metric on the quasilocal boundary $B$. The variations induced in the entropy, energy, and metric $\sigma_{a b}$ are variations in both the constant of integration $r_{\mathrm{H}}$ and the size of the quasilocal system $R$. Two unusual features of the CCBH spacetime thermodynamics are the facts that the entropy depends on the size $R$ of the quasilocal system and that the metric of the quasilocal boundary depends on the constant of integration $r_{\mathrm{H}}$. Thus, under variations in the parameter $r_{\mathrm{H}}$ alone, there is work done by the surface stress; similarly, a process involving a change in the size of the quasilocal system alone is not adiabatic.

We have shown that it is possible to compute the thermodynamic variables associated with the $\mathrm{CCBH}$ spacetime in $3+1$ dimensions as a solution to the theory of general relativity. In order to avoid the effects of the unusual asymptotic behavior of the spacetime, we have adopted quasilocal definitions of the thermodynamic variables. When the spacetime is foliated into leaves associated with the timelike Killing vector, the Euclidean instanton has an unusual topology: the foliation becomes degenerate on a cylinder that contains the bifurcation circle of the event horizon. The entropy is associated with the area of this cylinder, and it vanishes as the quasilocal surface approaches the horizon. The entropy is associated with the Killing horizon generated by the acceleration of static observers; such an entropy is also found for accelerating observers in a Rindler wedge of Minkowski spacetime [11]. We should emphasize that, unlike in Minkowski spacetime, a static observer is forced to see an acceleration horizon because of the black hole geometry. In 
addition, the metric on the quasilocal boundary depends on the constant of integration of the black hole solution. Because of this, it is difficult to find a reference spacetime that produces a suitable zero-point for the quasilocal energy. Nevertheless, the thermodynamic variables do satisfy the first law of thermodynamics given in Eq. (14).
We would like to thank Peter Peldán and Patrick Brady for their comments on an earlier draft of this paper. This work was supported in part by the Natural Sciences and Engineering Research Council of Canada. J. Creighton gratefully acknowledges partial support from NSF Grant No. AST-9417371.
[1] M. Bañados, Phys. Rev. D 57, 1068 (1998).

[2] M. Bañados, M. Henneaux, C. Teitelboim, and J. Zanelli, Phys. Rev. D 48, 1506 (1993).

[3] S. Holst and P. Peldán, Class. Quantum Grav. 14, 3433 (1997).

[4] S. Carlip, J. Gegenberg, and R. Mann, Phys. Rev. D 51, 6854 (1995).

[5] J. D. Brown, J. D. E. Creighton, and R. B. Mann, Phys. Rev. D 50, 6394 (1994); O. B. Zaslavskii, Class. Quantum Grav. 11, L33 (1994).
[6] D. Brill, J. Louko, and P. Peldán, Phys. Rev. D 56, 3600 (1997); L. Vanzo, ibid. 56, 6475 (1997).

[7] J. D. Brown and J. W. York, Phys. Rev. D 47, 1420 (1993).

[8] V. Iyer and R. M. Wald, Phys. Rev. D 52, 4430 (1995).

[9] R. M. Wald, Phys. Rev. D 48, 3427 (1993); V. Iyer and R. M. Wald, ibid. 50, 846 (1994).

[10] J. D. Brown and J. W. York, Phys. Rev. D 47, 1407 (1993).

[11] R. Laflamme, Phys. Lett. B 196, 449 (1987). 